\title{
Multilinear almost diagonal estimates and applications
}

\author{
by \\ ÁrpÁd BÉNYI and Nikolaos TzIRAKIs (Amherst, MA)
}

\begin{abstract}
We prove that an almost diagonal condition on the $(m+1)$-linear tensor associated to an $m$-linear operator implies boundedness of the operator on products of classical function spaces. We then provide applications to the study of certain singular integral operators.
\end{abstract}

1. Introduction. The study of multilinear operators has been motivated by their natural appearance in many aspects of harmonic analysis and partial differential equations. The multilinear point of view in the study of certain singular integrals, such as Calderón commutators, paraproducts or pseudodifferential operators, was pioneered and extensively pursued by Coifman and Meyer in the 1970s. Their ideas have had far reaching consequences and have led to an important development of the multilinear theory. We refer the reader to the work by Grafakos and Torres [8] and the references therein for more details about the more recent progress experienced in this theory.

One of the core ideas intensively used in the study of operators is to discretize them by decomposing the functions on which they act into elementary pieces. The study of the infinite-dimensional matrices obtained in this way usually yields information about the associated operators. This approach was carried out in the bilinear setting in [7]. There, the authors have quantified sufficient almost diagonal conditions on the corresponding trilinear discrete tensors that imply boundedness of the bilinear operators on two-folds of Lebesgue and other function spaces. The main results were then used to study the boundedness of certain bilinear pseudodifferential operators.

2000 Mathematics Subject Classification: Primary 47A07, 42B25, 42B35; Secondary 42B15, 42B20, 42C15, 42C40, 47G10, 47G30.

Key words and phrases: multilinear operators, almost diagonal conditions, wavelets, atoms, molecules, pseudodifferential operators, Calderón-Zygmund singular integrals, Triebel-Lizorkin spaces. 
The methods in [7] were inspired by the earlier work of Frazier and Jawerth [4] in the linear case. We wish to point out, however, that the bilinear almost diagonal estimates in [7] cannot be recovered from the linear ones by a mere freezing of one of the variables argument. As a simple mean to check the rather intricate bilinear almost diagonal estimates, the authors of [7] have introduced the notion of bilinear smooth molecule. Relying on this concept and certain atomic decomposition techniques, as well as a new notion of bilinear weak boundedness, Bényi has obtained in [1] a characterization of bilinear Calderón-Zygmund operators as being precisely those singular integral operators which map pairs of atoms into bilinear molecules. Using this fact, he has then established a reduced bilinear $T 1$ theorem in the context of Triebel-Lizorkin spaces.

Motivated by the previous works, we asked the following natural question in the $m$-linear setting, $m \geq 1$ :

Are there any sufficient conditions on the $(m+1)$-linear discrete tensor associated to an m-linear operator that imply continuity results on appropriate products of function spaces?

We answer this question in the affirmative in Theorem 1. Our approach is to first appropriately extend the definition of smooth molecules to the general $m$-linear setting, and then infer the corresponding $m$-linear version of the almost diagonal estimates on the tensor. These in turn will guarantee the boundedness of the operator defining the tensor. Our arguments will follow closely those in [7]. Nevertheless, in this general setting, some additional technical difficulties need to be overcome. In particular, a careful management of the combinatorics of the problem becomes very important.

In the last section we outline some applications of our results to the study of certain multilinear pseudodifferential operators, and discuss a reduced $m$-linear $T 1$ theorem in the context of Triebel-Lizorkin spaces.

2. General notation and preliminaries. We summarize here the common core of the notation used throughout this work, as well as some preliminary results. We will define the rest of the mathematical notation needed as it is introduced.

We will be working on the $n$-dimensional space $\mathbb{R}^{n}$. We let $\mathcal{D}=\mathcal{D}\left(\mathbb{R}^{n}\right)$ and $\mathcal{S}=\mathcal{S}\left(\mathbb{R}^{n}\right)$ be, respectively, the subspaces of $C^{\infty}=C^{\infty}\left(\mathbb{R}^{n}\right)$ of compactly supported functions and of Schwartz rapidly decreasing functions, with their usual topologies. Their duals are $\mathcal{D}^{\prime}=\mathcal{D}^{\prime}\left(\mathbb{R}^{n}\right)$, the set of all distributions on $\mathbb{R}^{n}$, and $\mathcal{S}^{\prime}=\mathcal{S}^{\prime}\left(\mathbb{R}^{n}\right)$, the set of all tempered distributions on $\mathbb{R}^{n}$. 
The Fourier transform of a tempered distribution $f$ in $n$-dimensional euclidean space is defined as

$$
\widehat{f}(\xi)=\int_{\mathbb{R}^{n}} f(x) e^{-i x \cdot \xi} d x
$$

The inverse Fourier transform is given by $\check{f}(\xi)=\widehat{f}(-\xi)$.

We denote by $L^{p}=L^{p}\left(\mathbb{R}^{n}\right)$ the classical Lebesgue spaces of measurable functions whose modulus to the $p$ th power is integrable, with the usual modification when $p=\infty$. For $0<p, s \leq \infty$ and arbitrary real $\alpha$, the homogeneous Triebel-Lizorkin spaces $\dot{F}_{p}^{\alpha, s}$ are defined by the quasi-norms

$$
\|f\|_{\dot{F}_{p}^{\alpha, s}}=\left\|\left(\sum_{\nu \in \mathbb{Z}}\left(2^{\nu \alpha}\left|f * \phi_{\nu}\right|\right)^{s}\right)^{1 / s}\right\|_{L^{p}\left(\mathbb{R}^{n}\right)},
$$

where $\phi \in \mathcal{S}$ is a fixed function such that $\widehat{\phi}(\xi)$ is supported in the annulus $1 / 2 \leq|\xi| \leq 2$ and $\widehat{\phi}(\xi)$ is bounded away from zero on the smaller annulus $3 / 5 \leq|\xi| \leq 5 / 3$. Here $\phi_{\nu}(x)=2^{\nu n} \phi\left(2^{\nu} x\right)$. In particular, for $\alpha=0$ and $s=2$, we recover the $L^{p}$ spaces, $L^{p} \approx \dot{F}_{p}^{0,2}$.

For $\nu \in \mathbb{Z}$ and $k=\left(k_{1}, \ldots, k_{n}\right) \in \mathbb{Z}^{n}$, let $Q_{\nu k}$ be the dyadic cube having the lower left corner $x_{\nu k}=2^{-\nu} k$ and side-length $l\left(Q_{\nu k}\right)=2^{-\nu}$,

$$
Q_{\nu k}=\left\{\left(x_{1}, \ldots, x_{n}\right) \in \mathbb{R}^{n}: 2^{-\nu} k_{i} \leq x_{i}<2^{-\nu}\left(k_{i}+1\right), 1 \leq i \leq n\right\} .
$$

We let $\chi_{Q_{\nu k}}$ denote the characteristic function of the dyadic cube and $\widetilde{\chi}_{Q_{\nu k}}=$ $2^{\nu n / 2} \chi_{Q_{\nu k}}$ its $L^{2}$ normalization. Define also

$$
\phi_{\nu k}(x)=2^{\nu n / 2} \phi\left(2^{\nu} x-k\right) .
$$

The Triebel-Lizorkin spaces admit almost orthogonal wavelet decompositions, that is, one can select a function $\phi$ such that for $f \in \dot{F}_{p}^{\alpha, s}$, $1<p, s<\infty$, we have

$$
f=\sum_{\nu, k}\left\langle f, \phi_{\nu k}\right\rangle \phi_{\nu k}
$$

The convergence of the series above is in $\dot{F}_{p}^{\alpha, s}$. Furthermore, the coefficients involved in the series (1) belong to appropriate spaces of sequences that characterize the Triebel-Lizorkin norm of $f$. More precisely (see [5, Ch. 5]),

$$
\|f\|_{\dot{F}_{p}^{\alpha, s}} \approx\left\|\left\{\left\langle f, \phi_{\nu k}\right\rangle\right\}\right\|_{\dot{f}_{p}^{\alpha, s}},
$$

where $\dot{f}_{p}^{\alpha, s}$ are spaces of sequences $\left\{s_{\nu k}\right\}$ with norms given by

$$
\left\|\left\{s_{\nu k}\right\}\right\|_{\dot{f}_{p}^{\alpha, s}}=\left\|\left(\sum_{\nu}\left(\sum_{k}\left|s_{\nu k}\right| 2^{\nu \alpha} \widetilde{\chi}_{Q_{\nu k}}\right)^{s}\right)^{1 / s}\right\|_{L^{p}\left(\mathbb{R}^{n}\right)} .
$$

The bilinear form $\langle\cdot, \cdot\rangle$ is the pairing between a distribution and a test function. The symbol $\approx$ refers to two positive quantities that have comparable size with constants independent of the parameters involved. 
The Triebel-Lizorkin spaces can also be characterized in terms of other basic objects, such as smooth atoms or molecules. For further details about Triebel-Lizorkin spaces as well as their relation to other classical function spaces, see Triebel's monograph [10], or the book of Frazier, Jawerth, and Weiss [5].

In this work we study $m$-linear operators $T: \mathcal{S} \times \cdots \times \mathcal{S} \rightarrow \mathcal{S}^{\prime}$ and their continuous extensions to various products of function spaces. These operators are linear in each entry and therefore have $m$ formal transposes. The $j$ th transpose $T^{* j}$ of $T$ is defined via

$$
\left\langle T^{* j}\left(f_{1}, \ldots, f_{m}\right), g\right\rangle=\left\langle T\left(f_{1}, \ldots, f_{j-1}, g, f_{j+1}, \ldots, f_{m}\right), f_{j}\right\rangle
$$

for all $f_{1}, \ldots, f_{m}, g$ in $\mathcal{S}$.

Let $T$ be a continuous $m$-linear operator from $\mathcal{S} \times \cdots \times \mathcal{S}$ into $\mathcal{S}^{\prime}$. A version of the Schwartz kernel theorem shows that any such $T$ must be given in the form

$$
\begin{array}{r}
T\left(f_{1}, \ldots, f_{m}\right)(x) \\
=\int_{\left(\mathbb{R}^{n}\right)^{m}} K\left(x, y_{1}, \ldots, y_{m}\right) f_{1}\left(y_{1}\right) \cdots f_{m}\left(y_{m}\right) d y_{1} \cdots d y_{m},
\end{array}
$$

where $K\left(x, y_{1}, \ldots, y_{m}\right)$ is a tempered distribution on $\left(\mathbb{R}^{n}\right)^{m+1}$ and the integral in (3) is interpreted in the sense of distributions, i.e.,

$$
\left\langle T\left(f_{1}, \ldots, f_{m}\right), g\right\rangle=\left\langle K, g \otimes f_{1} \otimes \cdots \otimes f_{m}\right\rangle .
$$

Here $g \otimes f_{1} \otimes \cdots \otimes f_{m}$ denotes the function

$$
\left(x, y_{1}, \ldots, y_{m}\right) \mapsto g(x) f_{1}\left(y_{1}\right) \cdots f_{m}\left(y_{m}\right) .
$$

One can easily check that if $T$ has the kernel $K$ then its $j$ th formal transpose $T^{* j}$ has the kernel $K^{* j}$ given by

$$
K^{* j}\left(x, y_{1}, \ldots, y_{m}\right)=K\left(y_{j}, y_{1}, \ldots, y_{j-1}, x, y_{j+1}, \ldots, y_{m}\right) .
$$

We will be interested in finding sufficient conditions to extend $m$-linear operators $T$ defined a priori on products of test functions to products of Triebel-Lizorkin spaces. We will do so in our main results, Theorem 1 and Theorem 2. In the proofs we will make use of the so-called vector-valued maximal theorem of Fefferman and Stein [3] that we state below.

Theorem A. Let $M$ denote the Hardy-Littlewood maximal operator on $\mathbb{R}^{n}$. Then, for $1<p<\infty$ and $1<s \leq \infty$, there exists a constant $C$ such that for all sequences $\left\{f_{j}\right\}$ of locally integrable functions,

$$
\left\|\left(\sum_{j} M\left(f_{j}\right)^{s}\right)^{1 / s}\right\|_{L^{p}\left(\mathbb{R}^{n}\right)} \leq C\left\|\left(\sum_{j}\left|f_{j}\right|^{s}\right)^{1 / s}\right\|_{L^{p}\left(\mathbb{R}^{n}\right)} .
$$


3. Multilinear molecules and almost diagonal conditions. Given an $m$-linear operator $T\left(f_{1}, \ldots, f_{m}\right)$, the associated $(m+1)$-linear discrete tensor $\tau(T)=\left\{\tau\left(\nu_{1}, l_{1}, \ldots, \nu_{m+1}, l_{m+1}\right)\right\}$ is the infinite array of numbers

$$
\tau\left(\nu_{1}, l_{1}, \ldots, \nu_{m+1}, l_{m+1}\right)=\left\langle T\left(\phi_{\nu_{1} l_{1}}, \ldots, \phi_{\nu_{m} l_{m}}\right), \phi_{\nu_{m+1} l_{m+1}}\right\rangle,
$$

where $\left\{\phi_{\nu l}\right\}$ is a fixed family of almost orthogonal wavelets, $\nu_{i} \in \mathbb{Z}$, and $l_{i} \in \mathbb{Z}^{n}$ for $i=1, \ldots, m+1$. Conversely, an $(m+1)$-linear discrete tensor $\tau$ defines an $m$-linear operator

$$
\begin{aligned}
& T\left(f_{1}, \ldots, f_{m}\right) \\
& \quad=\sum_{\nu_{i}, l_{i}} \tau\left(\nu_{1}, l_{1}, \ldots, \nu_{m+1}, l_{m+1}\right)\left\langle f_{1}, \phi_{\nu_{1} l_{1}}\right\rangle \cdots\left\langle f_{m}, \phi_{\nu_{m} l_{m}}\right\rangle \phi_{\nu_{m+1} l_{m+1}}
\end{aligned}
$$

for which the corresponding tensor is $\tau(T)=\tau$. It is important to observe that the wavelet representation (1) reduces the study of $m$-linear operators $T$ on products of Triebel-Lizorkin spaces $\dot{F}_{p_{1}, s_{1}}^{\alpha_{1}} \times \cdots \times \dot{F}_{p_{m}}^{\alpha_{m}, s_{m}}$ to the study of tensors $\tau(T)$ on products of spaces of sequences $\dot{f}_{p_{1}}^{\alpha_{1}, s_{1}} \times \cdots \times \dot{f}_{p_{m}}^{\alpha_{m}, s_{m}}$.

We now introduce the notion of $m$-linear smooth molecule by extending the definition given in [7] in the case $m=2$.

Let $K \in \mathbb{Z}_{+}$. A collection of functions $\left\{\psi_{\mu_{1}, l_{1}, \ldots, \mu_{m}, l_{m}}\right\}=\left\{\psi_{\left(\mu_{i}, l_{i}\right)}\right\}$, where $\mu_{i} \in \mathbb{Z}$ and $l_{i} \in \mathbb{Z}^{n}$ for $i=1, \ldots, m$, is called a family of $m$-linear $K$-smooth molecules if for all integers $N>n$ and all multi-indices $\gamma$ with $|\gamma| \leq K$, there exist constants $C_{N, \gamma}$ such that

$$
\left|D^{\gamma} \psi_{\left(\mu_{i}, l_{i}\right)}(x)\right| \leq C_{N, \gamma} \frac{2^{(n / 2) \sum_{i} \mu_{i}} \max _{i} 2^{\mu_{i}|\gamma|}}{\prod_{i}\left(1+2^{\mu_{i}}\left|x-2^{-\mu_{i}} l_{i}\right|\right)^{N}}
$$

and

$$
\int \psi_{\left(\mu_{i}, l_{i}\right)}(x) d x=0
$$

for all $\mu_{i}, l_{i}, i=1, \ldots, m$.

To emphasize the importance of this concept for our discussion, we recall that bilinear operators that map pairs of wavelets into bilinear smooth molecules are known to be bounded. The following theorem was proved in [7].

THEOREM B. Let $T$ be a bilinear operator so that for a wavelet family $\left\{\phi_{\mu l}\right\}$ the three families $\left\{T\left(\phi_{\mu_{1} l_{1}}, \phi_{\mu_{2} l_{2}}\right)\right\},\left\{T^{* 1}\left(\phi_{\mu_{1} l_{1}}, \phi_{\mu_{2} l_{2}}\right)\right\}$, and $\left\{T^{* 2}\left(\phi_{\mu_{1} l_{1}}, \phi_{\mu_{2} l_{2}}\right)\right\}$ are families of bilinear 1 -smooth molecules. Then $T$ can be extended as a bounded operator from $\dot{F}_{p}^{0, s_{1}} \times \dot{F}_{q}^{0, s_{2}}$ into $\dot{F}_{r}^{0, s_{3}}$, where $1<p, q, r<\infty, 1 / p+1 / q=1 / r, 1<s_{1}, s_{2} \leq \infty, 1 \leq s_{3}<\infty$ and $1 / s_{1}+1 / s_{2}=1 / s_{3}$.

Theorem B is implied by a more general result, which states that a bilinear operator is bounded provided that certain bilinear almost diagonal conditions on the associated trilinear discrete tensor hold. We have (see [7]): 
TheOREM C. Suppose that the tensor $\left\{\tau\left(\mu_{1}, l_{1} ; \mu_{2}, l_{2} ; \mu_{3}, l_{3}\right)\right\}$ associated to a bilinear operator $T$ satisfies the almost diagonal estimates

$$
\begin{aligned}
& \left|\tau\left(\mu_{1}, l_{1}, \mu_{2}, l_{2}, \mu_{3}, l_{3}\right)\right| \\
& \leq \frac{C \cdot 2^{-\left(\max _{i} \mu_{i}-\min _{i} \mu_{i}\right) \varepsilon} 2^{-(n / 2) \max _{i} \mu_{i}} 2^{(n / 2) \operatorname{med}_{i} \mu_{i}} 2^{(n / 2) \min _{i} \mu_{i}}}{\left(P\left(\mu_{1}, l_{1}, \mu_{2}, l_{2}, \mu_{3}, l_{3}\right)\right)^{N}}
\end{aligned}
$$

for some $C>0, N>n$ and $\varepsilon>0$, where

$$
\begin{aligned}
& P\left(\mu_{1}, l_{1}, \mu_{2}, l_{2}, \mu_{3}, l_{3}\right)=\left(1+2^{\min \left(\mu_{1}, \mu_{2}\right)}\left|2^{-\mu_{1}} l_{1}-2^{-\mu_{2}} l_{2}\right|\right) \\
& \quad \times\left(1+2^{\min \left(\mu_{2}, \mu_{3}\right)}\left|2^{-\mu_{2}} l_{2}-2^{-\mu_{3}} l_{3}\right|\right)\left(1+2^{\min \left(\mu_{3}, \mu_{1}\right)}\left|2^{-\mu_{3}} l_{3}-2^{-\mu_{1}} l_{1}\right|\right) .
\end{aligned}
$$

Then the corresponding operator $T$ can be extended to be bounded from $\dot{F}_{p}^{0, s_{1}} \times \dot{F}_{q}^{0, s_{2}}$ into $\dot{F}_{r}^{0, s_{3}}$, where $1<p, q, r<\infty, 1 / p+1 / q=1 / r$, $1<s_{1}, s_{2} \leq \infty, 1 \leq s_{3}<\infty$ and $1 / s_{1}+1 / s_{2}=1 / s_{3}$.

For three real numbers $a, b, c$ such that $a \leq b \leq c$, the medium number is defined as

$$
\operatorname{med}(a, b, c)=b .
$$

Under the hypothesis of Theorem B, it is easy to check that the associated tensor $\tau$ satisfies the bilinear almost diagonal estimates in Theorem $\mathrm{C}$ and the conclusion of the former theorem follows immediately.

Let us make some further comments about the statements of the theorems above. Granted one agrees to our definition of a multilinear smooth molecule, generalizing the statement of Theorem B is an easy task. However, one should not forget that Theorem B is a consequence of Theorem $\mathrm{C}$, and we do not know just yet what the $m$-linear versions of the almost diagonal estimates look like. Moreover, it is not completely obvious how to generalize the bilinear estimates to get, say, the trilinear ones. By this we mean that the statement in the case $m=2$ solely is not conclusive about the outlook of the general estimates. For example, should one worry about defining the medium number of four or more real numbers? Or, how many terms are needed in general when dealing with the multilinear version of the product $P$ above? It seems reasonable to believe that to extend the previous results properly one should examine more closely how the bilinear almost diagonal conditions arise in the first place. The goal of the three lemmas below is to provide an explanation of the following

Claim. The proper m-linear almost diagonal estimates satisfied by the $(m+1)$-linear discrete tensor associated with an $m$-linear operator $T$ should be of the following form:

$$
\begin{aligned}
& \left|\tau\left(\mu_{1}, l_{1}, \ldots, \mu_{m+1}, l_{m+1}\right)\right| \\
& \leq \frac{C \cdot 2^{-\left(\max _{i} \mu_{i}-\min _{i} \mu_{i}\right) \varepsilon} 2^{-(n / 2) \max _{i} \mu_{i}} 2^{(n / 2) \sum_{i \neq i_{0}} \mu_{i}}}{\prod_{1 \leq i<j \leq m+1}\left(1+2^{\min \left(\mu_{i}, \mu_{j}\right)}\left|2^{-\mu_{i}} l_{i}-2^{-\mu_{j}} l_{j}\right|\right)^{N}}
\end{aligned}
$$


for some $C>0, N>n, \varepsilon>0$, where $i_{0}$ denotes the index for which $\mu_{i_{0}}=\max _{i} \mu_{i}$.

A first convincing indication that our claim is correct is provided by the estimates on integrals of products of $m+1$ wavelets. We have:

LEMma 1. Suppose that the functions $\phi_{\mu_{i}}$ defined on $\mathbb{R}^{n}$ satisfy, for all $x \in \mathbb{R}^{n}, N>n$, and some $x_{\mu_{i}}$ and $C_{N}>0$, the following estimates:

$$
\left|\phi_{\mu_{i}}(x)\right| \leq C_{N} \frac{2^{(n / 2) \mu_{i}}}{\left(1+2^{\mu_{i}}\left|x-x_{\mu_{i}}\right|\right)^{N}}, \quad i=1, \ldots, m+1 .
$$

Then

$$
\int_{\mathbb{R}^{n}} \prod_{i=1}^{m+1}\left|\phi_{\mu_{i}}(x)\right| d x \leq \frac{C_{N} \cdot 2^{-(n / 2) \max _{i} \mu_{i}} 2^{(n / 2) \sum_{i \neq i_{0}} \mu_{i}}}{\prod_{1 \leq i<j \leq m+1}\left(1+2^{\min \left(\mu_{i}, \mu_{j}\right)}\left|x_{\mu_{i}}-x_{\mu_{j}}\right|\right)^{N}} .
$$

Proof. The proof is adapted from its bilinear counterpart in [7]. For the sake of completeness, we outline the main points of the argument here. Due to symmetry and a suitable change of variables we may assume that $\mu_{1} \geq \cdots \geq \mu_{m} \geq \mu_{m+1}=0$ and $x_{\mu_{m+1}}=0$. In this case, we can bound the integral on the left hand side of (10) by a suitable constant times the integral

$$
I=\int_{\mathbb{R}^{n}} \prod_{i=1}^{m} \frac{2^{(n / 2) \mu_{i}}}{\left(1+2^{\mu_{i}}\left|x-x_{\mu_{i}}\right|\right)^{M}} \cdot \frac{1}{(1+|x|)^{M}} d x,
$$

where $M$ is some integer larger than $m N$. A convenient choice of $M$ will be given later. Note that

$$
I=2^{(n / 2) \sum_{i=1}^{m} \mu_{i}} \sum_{i=1}^{m} \sum_{t_{i}=0}^{\infty} 2^{-M \sum_{i=1}^{m} t_{i}} \int_{R_{t_{1}}, \ldots, t_{m}} \frac{1}{(1+|x|)^{M}} d x .
$$

Here, the region $R_{t_{1}, \ldots, t_{m}}$ of integration is given by

$$
R_{t_{1}, \ldots, t_{m}}=\left\{x \in \mathbb{R}^{n}:\left|x-x_{\mu_{i}}\right| \sim 2^{t_{i}-\mu_{i}} \text { for all } 1 \leq i \leq m\right\}
$$

and $\left|x-x_{\mu}\right| \sim 2^{t-\mu}$ means $2^{t-\mu} \leq\left|x-x_{\mu}\right| \leq 2^{t+1-\mu}$ for $t \geq 1$, and $\left|x-x_{\mu}\right| \leq 2^{-\mu}$ for $t=0$. Next, observe that, as long as $R_{t_{1}, \ldots, t_{m}}$ is nonempty, for $1 \leq i<j \leq m$ we have

$$
1+2^{\mu_{i}}\left|x_{\mu_{i}}-x_{\mu_{j}}\right| \leq 8 \max \left(2^{t_{i}}, 2^{t_{j}}\right)
$$

while for $2 \leq i \leq m$ we can write

$$
1+\left|x_{\mu_{i}}\right| \leq 8\left(1+\left|x_{\mu_{1}}\right|\right) \max \left(2^{t_{1}}, 2^{t_{i}}\right) .
$$

Due to the choice of $M$, and the estimates (12) and (13) above, from Lemma 1 in [7] we get the following sequence of inequalities: 


$$
\begin{aligned}
\int_{R_{t_{1}, \ldots, t_{m}}} & \frac{1}{(1+|x|)^{M}} d x \leq \frac{C \max \left(2^{\left(t_{1}-\mu_{1}\right) n}, 2^{\left(t_{1}-\mu_{1}\right) m N}\right)}{\left(1+\left|x_{\mu_{1}}\right|\right)^{m N}} \\
\leq & \frac{C \max \left(2^{\left(t_{1}-\mu_{1}\right) n}, 2^{\left(t_{1}-\mu_{1}\right) m N}\right)}{\prod_{i=1}^{m}\left(1+\left|x_{\mu_{i}}\right|\right)^{N}} \prod_{i=2}^{m}\left(\max \left(2^{t_{1}}, 2^{t_{i}}\right)\right)^{N} \\
\leq & \frac{C \max \left(2^{\left(t_{1}-\mu_{1}\right) n}, 2^{\left(t_{1}-\mu_{1}\right) m N}\right) \prod_{1 \leq i<j \leq m}\left(\max \left(2^{t_{i}}, 2^{t_{j}}\right)\right)^{l(i) N}}{\prod_{i=1}^{m}\left(1+\left|x_{\mu_{i}}\right|\right)^{N} \prod_{1 \leq i<j \leq m}\left(1+2^{\min \left(\mu_{i}, \mu_{j}\right)}\left|x_{\mu_{i}}-x_{\mu_{j}}\right|\right)^{N}}
\end{aligned}
$$

where $l(i)=2$ if $i=1$, and $l(i)=1$ if $2 \leq i \leq m$. Going back to the integral $I$, we note that we can bound it by

$$
\begin{aligned}
I \leq & C \cdot 2^{(n / 2) \sum_{i=1}^{m} \mu_{i}} \sum_{i=1}^{m} \sum_{t_{i}=0}^{\infty} 2^{-M \sum_{i=1}^{m} t_{i}} \\
& \times \frac{\max \left(2^{\left(t_{1}-\mu_{1}\right) n}, 2^{\left(t_{1}-\mu_{1}\right) m N}\right) \prod_{1 \leq i<j \leq m}\left(\max \left(2^{t_{i}}, 2^{t_{j}}\right)\right)^{l(i) N}}{\prod_{i=1}^{m}\left(1+\left|x_{\mu_{i}}\right|\right)^{N} \prod_{1 \leq i<j \leq m}\left(1+2^{\min \left(\mu_{i}, \mu_{j}\right)}\left|x_{\mu_{i}}-x_{\mu_{j}}\right|\right)^{N}}
\end{aligned}
$$

If we now choose $M=(3 m-1) N$ and the constant $C=C_{N}$ appropriately, then the last geometric series converges and we can estimate $I$ by

$$
\frac{C_{N} \cdot 2^{-(n / 2) \mu_{1}} 2^{(n / 2) \sum_{i=2}^{m} \mu_{i}}}{\prod_{i=1}^{m}\left(1+\left|x_{\mu_{i}}\right|\right)^{N} \prod_{1 \leq i<j \leq m}\left(1+2^{\min \left(\mu_{i}, \mu_{j}\right)}\left|x_{\mu_{i}}-x_{\mu_{j}}\right|\right)^{N}},
$$

which proves (10).

REMARK 1. If we pick $x_{\mu_{i}}=2^{-\mu_{i}} l_{i}$ and $\phi_{\mu_{i}}=\phi_{\mu_{i} l_{i}}$ with $\phi$ as in the almost orthogonal wavelet decomposition (1), then it is easy to see that condition (9) is satisfied. Moreover, we have

$$
\left|D^{\gamma} \phi_{\mu_{i} l_{i}}\right| \leq C_{N, \gamma} \frac{2^{(n / 2) \mu_{i}} 2^{|\gamma| \mu_{i}}}{\left(1+2^{\mu_{i}}\left|x-2^{-\mu_{i}} l_{i}\right|\right)^{N}},
$$

for all multiindices $\gamma$ and all positive integers $N$. Note also that in this case there is a strong resemblance of the right hand sides of the inequalities (8) and (10).

The estimate of the integral of a product of wavelets does not explain, however, the appearance of the term $2^{-\left(\max _{i} \mu_{i}-\min _{i} \mu_{i}\right) \varepsilon}$ in $(8)$. Let us assume for the moment that if a multilinear operator $T$ maps wavelets into multilinear molecules,

$$
T\left(\phi_{\mu_{1} l_{1}}, \ldots, \phi_{\mu_{m} l_{m}}\right)=\psi_{\left(\mu_{i}, l_{i}\right)},
$$

then an estimate like (8) holds on the tensor $\tau$ defined by (5). This suggests that, before moving any further, one should examine the interaction between a wavelet and a multilinear molecule. This analysis yields the results outlined in the following two lemmas. It shows, in particular, why our previous 
assumption is true. For a proof of these lemmas in the case $m=2$, see [7]. The details of the proof in the general case are left to the interested reader.

LEMMA 2. Let $\phi_{\mu_{m+1} l_{m+1}}$ be a wavelet and $\psi_{\mu_{1}, l_{1}, \ldots, \mu_{m}, l_{m}}$ an m-linear 0 -smooth molecule such that $\max _{1 \leq i \leq m} \mu_{i} \geq \mu_{m+1}$. Then for all positive integers $N>n$ there exists a positive constant $C_{N}$ such that

$$
\begin{aligned}
& \left|\left\langle\psi_{\mu_{1}, l_{1}, \ldots, \mu_{m}, l_{m}}, \phi_{\mu_{m+1} l_{m+1}}\right\rangle\right| \\
& \quad \leq \frac{C_{N} \cdot 2^{-\left(\max _{1 \leq i \leq m} \mu_{i}-\mu_{m+1}\right)} 2^{-(n / 2) \max _{1 \leq i \leq m} \mu_{i}} 2^{(n / 2) \sum_{i=1, i \neq i_{0}}^{m+1} \mu_{i}}}{\prod_{1 \leq i<j \leq m+1}\left(1+2^{\min \left(\mu_{i}, \mu_{j}\right)}\left|2^{-\mu_{i}} l_{i}-2^{-\mu_{j}} l_{j}\right|\right)^{N}},
\end{aligned}
$$

where $i_{0}$ denotes the index for which $\mu_{i_{0}}=\max _{1 \leq i \leq m} \mu_{i}$.

LEMMA 3. Let $\phi_{\mu_{m+1} l_{m+1}}$ be a wavelet and $\psi_{\mu_{1}, l_{1}, \ldots, \mu_{m}, l_{m}}$ an m-linear 1 -smooth molecule such that $\max _{1 \leq i \leq m} \mu_{i} \leq \mu_{m+1}$. Then for all positive integers $N>n$ there exists a positive constant $C_{N}$ such that

$$
\begin{aligned}
\mid\left\langle\psi_{\mu_{1}, l_{1}, \ldots, \mu_{m}, l_{m}},\right. & \left.\phi_{\mu_{m+1} l_{m+1}}\right\rangle \mid \\
& \leq \frac{C_{N} \cdot 2^{-\left(\mu_{m+1}-\max _{1 \leq i \leq m} \mu_{i}\right)} 2^{-(n / 2) \mu_{m+1}} 2^{(n / 2) \sum_{i=1}^{m} \mu_{i}}}{\prod_{1 \leq i<j \leq m+1}\left(1+2^{\min \left(\mu_{i}, \mu_{j}\right)}\left|2^{-\mu_{i}} l_{i}-2^{-\mu_{j}} l_{j}\right|\right)^{N}} .
\end{aligned}
$$

4. Boundedness of multilinear operators. The main results are stated and proved below.

TheOREM 1. Suppose that the $(m+1)$-linear discrete tensor $\left\{\tau\left(\mu_{1}, l_{1}, \ldots\right.\right.$ $\left.\left.\ldots, \mu_{m+1}, l_{m+1}\right)\right\}$ associated to an m-linear operator $T$ satisfies the almost diagonal estimates (8). Then $T$ can be extended as a bounded operator from $\dot{F}_{p_{1}}^{0, s_{1}} \times \cdots \times \dot{F}_{p_{m}}^{0, s_{m}}$ into $\dot{F}_{p}^{0, s}$ for $1<p, p_{1}, \ldots, p_{m}<\infty, 1 / p_{1}+\cdots+1 / p_{m}$ $=1 / p, 1 \leq s<\infty, 1<s_{1}, \ldots, s_{m} \leq \infty$ and $1 / s_{1}+\cdots+1 / s_{m}=1 / s$.

Proof. If $\left\{\phi_{\mu l}\right\}$ is a fixed family of wavelets, for $f_{1}, \ldots, f_{m} \in \mathcal{S}$ we can write

$$
\begin{aligned}
T & \left(f_{1}, \ldots, f_{m}\right) \\
& =\sum_{\mu_{i}, l_{i}} \tau\left(\mu_{1}, l_{1}, \ldots, \mu_{m+1}, l_{m+1}\right)\left\langle f_{1}, \phi_{\mu_{1} l_{1}}\right\rangle \cdots\left\langle f_{m}, \phi_{\mu_{m} l_{m}}\right\rangle \phi_{\mu_{m+1} l_{m+1}} \\
& =\sum_{\mu_{m+1}, l_{m+1}} S_{\mu_{m+1} l_{m+1}} \phi_{\mu_{m+1} l_{m+1}},
\end{aligned}
$$

where

$$
S_{\mu_{m+1} l_{m+1}}=\sum_{\mu_{i}, l_{i}, i \neq m+1} \tau\left(\mu_{1}, l_{1}, \ldots, \mu_{m+1}, l_{m+1}\right)\left\langle f_{1}, \phi_{\mu_{1} l_{1}}\right\rangle \cdots\left\langle f_{m}, \phi_{\mu_{m} l_{m}}\right\rangle .
$$


Let $v_{\mu_{i} l_{i}}=\left\langle f_{i}, \phi_{\mu_{i} l_{i}}\right\rangle$. Note that

$$
\left|S_{\mu_{m+1} l_{m+1}}\right| \leq \sum_{\pi \in \mathcal{P}_{m+1}}\left|S_{\mu_{m+1} l_{m+1}}^{(\pi)}\right|
$$

where $\mathcal{P}_{m+1}$ denotes the set of all permutations of $\{1, \ldots, m+1\}$ and

$$
S_{\mu_{m+1} l_{m+1}}^{(\pi)}=\sum_{\substack{\mu_{\pi(1)} \leq \cdots \leq \mu_{\pi(m+1)} \\ l_{i}, 1 \leq i \leq m}} \tau\left(\mu_{1}, l_{1}, \ldots, \mu_{m+1}, l_{m+1}\right) v_{\mu_{1} l_{1}} \cdots v_{\mu_{m} l_{m}} .
$$

Furthermore, using the wavelet characterization of Triebel-Lizorkin spaces (2), we have

$$
\begin{aligned}
& \left\|T\left(f_{1}, \ldots, f_{m}\right)\right\|_{\dot{F}_{p}^{0, s}} \\
& \quad \leq C \sum_{\pi \in \mathcal{P}_{m+1}}\left\|\left(\sum_{\mu_{m+1}}\left(\sum_{l_{m+1}}\left|S_{\mu_{m+1} l_{m+1}}^{(\pi)}\right| \widetilde{\chi}_{Q_{\mu_{m+1} l_{m+1}}}\right)^{s}\right)^{1 / s}\right\|_{L^{p}} .
\end{aligned}
$$

Our goal is to estimate the sums inside the $L^{p}$ norms on the right hand side of (15). In order to do so, we will first estimate appropriately the terms $S_{\mu_{m+1} l_{m+1}}^{(\pi)}$ and then use the vector-valued maximal inequality stated in Theorem A.

Let us consider first the term $S_{\mu_{m+1} l_{m+1}}^{(\pi)}$ in which $\pi=$ Id represents the identity permutation, $\pi(i)=i, 1 \leq i \leq m+1$; that is, we would like to find a bound on

$$
S_{\mu_{m+1} l_{m+1}}^{(\mathrm{Id})}=\sum_{\substack{\mu_{1} \leq \cdots \leq \mu_{m+1} \\ l_{1}, \ldots, l_{m}}} \tau\left(\mu_{1}, l_{1}, \ldots, \mu_{m+1}, l_{m+1}\right) v_{\mu_{1} l_{1}} \cdots v_{\mu_{m} l_{m}} .
$$

The almost diagonal condition (8) implies

$$
\begin{aligned}
& \left|\tau\left(\mu_{1}, l_{1}, \ldots, \mu_{m+1}, l_{m+1}\right)\right| \\
& \quad \leq \frac{C_{N} \cdot 2^{-\left(\mu_{m+1}-\mu_{1}\right) \varepsilon} 2^{-(n / 2) \mu_{m+1}} 2^{(n / 2) \sum_{i=1}^{m} \mu_{i}}}{\prod_{1 \leq i<j \leq m+1}\left(1+2^{\min \left(\mu_{i}, \mu_{j}\right)}\left|2^{-\mu_{i}} l_{i}-2^{-\mu_{j}} l_{j}\right|\right)^{N}} .
\end{aligned}
$$

From (16) we immediately obtain the following bound on $S_{\mu_{m+1} l_{m+1}}^{(\mathrm{Id})}$ :

$$
\begin{aligned}
C_{N} & \sum_{\mu_{1} \leq \cdots \leq \mu_{m+1}} \sum_{k=1}^{m} \sum_{i_{k}=0}^{\infty} \sum_{l_{1}, \ldots, l_{m}} \\
& \times \frac{2^{-\left(\mu_{m+1}-\mu_{1}\right) \varepsilon} 2^{-(n / 2) \mu_{m+1}} 2^{(n / 2) \sum_{i=1}^{m} \mu_{i}} \prod_{k=1}^{m} 2^{-i_{k} N}}{\prod_{1 \leq i<j \leq m}\left(1+2^{\min \left(\mu_{i}, \mu_{j}\right)}\left|2^{-\mu_{i}} l_{i}-2^{-\mu_{j}} l_{j}\right|\right)^{N}} \prod_{i=1}^{m}\left|v_{\mu_{i} l_{i}}\right|,
\end{aligned}
$$

where the indices $i_{k}, 1 \leq k \leq m$, range over the sets in $\mathbb{Z}^{n}$ for which $2^{\mu_{k}}\left|2^{-\mu_{k}} l_{k}-2^{-\mu_{m+1}} l_{m+1}\right| \sim 2^{i_{k}}$. Let

$$
M^{\mu_{i}}=M\left(\sum_{l_{i}}\left|v_{\mu_{i} l_{i}}\right| \widetilde{\chi}_{Q_{\mu_{i} l_{i}}}\right),
$$

where $M$ denotes the Hardy-Littlewood maximal function. 
If we now use (17), a similar argument to one in [7] will show that we can bound $S_{\mu_{m+1} l_{m+1}}^{(\mathrm{Id})}$ by a constant (depending on $N$ ) multiple of

$$
\begin{aligned}
\sum_{\mu_{1} \leq \cdots \leq \mu_{m+1}} \sum_{k=1}^{m} \sum_{i_{k}=0}^{\infty} 2^{-\left(\mu_{m+1}-\mu_{1}\right) \varepsilon} 2^{-(n / 2) \mu_{m+1}} 2^{(n / 2) \sum_{i=1}^{m} \mu_{i}} \\
\times \prod_{k=1}^{m} 2^{-i_{k} N} 2^{(n / 2) \mu_{k}} \prod_{k=1}^{m} 2^{\left(i_{k}-\mu_{k}\right) n} M^{\mu_{k}}(x)
\end{aligned}
$$

for all $x \in Q_{\mu_{m+1} l_{m+1}}$ (for the definition of these dyadic cubes and the rest of the notation, see again Section 2). By choosing $N>n$ conveniently (so that we can sum the infinite series over the indices $i_{k}, 1 \leq k \leq m$ ), we see that the latter inequality implies

$$
\left|S_{\mu_{m+1} l_{m+1}}^{(\mathrm{Id})} \tilde{\chi}_{Q_{\mu_{m+1} l_{m+1}}}\right| \leq C \sum_{\mu_{1} \leq \cdots \leq \mu_{m+1}} 2^{-\left(\mu_{m+1}-\mu_{1}\right) \varepsilon} \prod_{k=1}^{m} M^{\mu_{k}} .
$$

This immediately yields the following sequence of inequalities:

$$
\begin{aligned}
& \left(\sum_{\mu_{m+1}}\left(\sum_{l_{m+1}}\left|S_{\mu_{m+1} l_{m+1}}^{\mathrm{IId})}\right| \widetilde{\chi}_{Q_{\mu_{m+1} l_{m+1}}}\right)^{s}\right)^{1 / s} \\
& \leq C\left(\sum_{\mu_{m+1}}\left(\sum_{\mu_{m} \leq \mu_{m+1}} \ldots \sum_{\mu_{1} \leq \mu_{2}} \prod_{j=2}^{m+1} 2^{-\left(\mu_{j}-\mu_{1}\right) \varepsilon / m} \prod_{k=1}^{m} M^{\mu_{k}}\right)^{s}\right)^{1 / s} \\
& =C\left\|\sum_{\mu_{m} \leq \mu_{m+1}} \ldots \sum_{\mu_{1} \leq \mu_{2}} \prod_{j=2}^{m+1} 2^{-\left(\mu_{j}-\mu_{1}\right) \varepsilon / m} \prod_{k=1}^{m} M^{\mu_{k}}\right\|_{l^{s}\left(\mu_{m+1}\right)} \\
& \leq C\left\|\prod_{j=2}^{m} 2^{-\left(\mu_{j}-\mu_{1}\right) \varepsilon / m} \chi_{\left\{\mu_{j-1} \leq \mu_{j}\right\}} \prod_{k=1}^{m} M^{\mu_{k}}\right\|_{l^{s}\left(\mu_{1}, \ldots, \mu_{m}\right)} \\
& \leq C \prod_{k=1}^{m} \|\left\{M^{\left.\mu_{k}\right\} \|_{l^{s} k\left(\mu_{k}\right)} .}\right.
\end{aligned}
$$

In the transition from the third to the fourth line we used the fact that the operator in question is of convolution type with the $l^{1}$ kernel

$$
K\left(\mu_{m+1}, \mu_{1}, \ldots, \mu_{m}\right)=2^{-\left(\mu_{m+1}-\mu_{1}\right) \varepsilon / m} \prod_{j=2}^{m} \chi_{\left\{\mu_{j-1} \leq \mu_{j}\right\}} .
$$

In the transition from the fourth to the last line we used Hölder's inequality with $1 / s_{1}+\cdots+1 / s_{m}=1 / s$.

Take $L^{p}$ norms in (19) and apply Hölder's inequality with $1 / p=1 / p_{1}+$ $\cdots+1 / p_{m}$ to obtain 


$$
\begin{array}{r}
\left\|\left(\sum_{\mu_{m+1}}\left(\sum_{l_{m+1}}\left|S_{\mu_{m+1} l_{m+1}}^{(\mathrm{Id})}\right| \tilde{\chi}_{Q_{\mu_{m+1} l_{m+1}}}\right)^{s}\right)^{1 / s}\right\|_{L^{p}} \\
\leq C \prod_{k=1}^{m}\left\|\left(\sum_{\mu_{k}}\left(M^{\mu_{k}}\right)^{s_{k}}\right)^{1 / s_{k}}\right\|_{L^{p_{k}}} .
\end{array}
$$

If we recall the definition (18) of $M^{\mu_{k}}$, and use Theorem A and once again the wavelet characterization of Triebel-Lizorkin spaces, we conclude from (20) that

$$
\begin{aligned}
\left\|\left(\sum_{\mu_{m+1}}\left(\sum_{l_{m+1}}\left|S_{\mu_{m+1} l_{m+1}}^{(\mathrm{Id})}\right| \widetilde{\chi}_{Q_{\mu_{m+1} l_{m+1}}}\right)^{s}\right)^{1 / s}\right\|_{L^{p}} \\
\leq C \prod_{k=1}^{m}\left\|\left\{\left\langle f_{k}, \phi_{\mu_{k} l_{k}}\right\rangle\right\}\right\|_{\dot{f}_{p_{k}}^{0, s_{k}}} \leq C \prod_{k=1}^{m}\left\|f_{k}\right\|_{\dot{F}_{p_{k}}^{0, s_{k}}} .
\end{aligned}
$$

To finish up the proof, we use (21) to bound all the sums appearing on the right hand side of (15). We start by observing that if a tensor $\tau$ is bounded from $\dot{f}_{p_{1}}^{0, s_{1}} \times \cdots \times \dot{f}_{p_{m}}^{0, s_{m}}$ into $\dot{f}_{p}^{0, s}$ with $1 / p_{1}+\cdots+1 / p_{m}=1 / p$, $1 / s_{1}+\cdots+1 / s_{m}=1 / s$, then the $j$ th formal transpose $\tau^{* j}$ is bounded from $\dot{f}_{p_{1}}^{0, s_{1}} \times \cdots \times \dot{f}_{p_{j-1}}^{0, s_{j-1}} \times \dot{f}_{p^{\prime}}^{0, s^{\prime}} \times \dot{f}_{p_{j+1}}^{0, s_{j+1}} \times \cdots \times \dot{f}_{p_{m}}^{0, s_{m}}$ into $\dot{f}_{p_{j}^{\prime}}^{0, s_{j}^{\prime}}$, where the indices now satisfy $1 / p_{1}+\cdots+1 / p_{j-1}+1 / p^{\prime}+1 / p_{j+1}+\cdots+1 / p_{m}=1 / p_{j}^{\prime}$ and $1 / s_{1}+\cdots+1 / s_{j-1}+1 / s^{\prime}+1 / s_{j+1}+\cdots+1 / s_{m}=1 / s_{j}$. By interchanging the roles of the indices $\mu_{1}, \ldots, \mu_{m}$ (that is, by looking at all the permutations in $\mathcal{P}_{m}$ as elements of $\mathcal{P}_{m+1}$ with the last component fixed) we obtain the same bound as in (21) for a number of $m$ ! of these sums. The same argument applied to each of the $m$ formal transposes of the tensor $\tau$ (which amounts to composing permutations in $\mathcal{P}_{m}$ with transpositions in $\mathcal{P}_{m+1}$ ) gives us the bounds for another $m \cdot m$ ! of the sums, to a total of $m !+m \cdot m !=(m+1)$ ! distinct sums of the form $S_{\mu_{m+1} l_{m+1}}^{(\pi)}, \pi \in \mathcal{P}_{m+1}$. Since the cardinality of $\mathcal{P}_{m+1}$ is precisely $(m+1)$ !, we are done.

THEOREM 2. Let $T$ be an m-linear operator so that for a wavelet family $\left\{\phi_{\mu l}\right\}$ the families $\left\{T\left(\phi_{\mu_{1} l_{1}}, \ldots, \phi_{\mu_{m} l_{m}}\right)\right\}$ and $\left\{T^{* i}\left(\phi_{\mu_{1} l_{1}}, \ldots, \phi_{\mu_{m} l_{m}}\right)\right\}$, $i=1, \ldots, m$, are families of $m$-linear 1 -smooth molecules. Then $T$ can be extended as a bounded operator from $\dot{F}_{p_{1}}^{0, s_{1}} \times \cdots \times \dot{F}_{p_{m}}^{0, s_{m}}$ into $\dot{F}_{p}^{0, s}$ for $1<$ $p, p_{1}, \ldots, p_{m}<\infty, 1 / p_{1}+\cdots+1 / p_{m}=1 / p, 1 \leq s<\infty, 1<s_{1}, \ldots, s_{m} \leq \infty$ and $1 / s_{1}+\cdots+1 / s_{m}=1 / s$.

Proof. This follows immediately from Theorem 1 and Lemmas 2 and 3.

REMARK 2. If we let $s_{i}=2$ for all $1 \leq i \leq m$, we see that under the hypothesis of either Theorem 1 or Theorem 2 , the $m$-linear operator $T$ can be extended to be bounded from $L^{p_{1}} \times \cdots \times L^{p_{m}}$ into $\dot{F}_{p}^{0,2 / m}$. But $\dot{F}_{p}^{0,2 / m} \hookrightarrow \dot{F}_{p}^{0,2}$ for $m \geq 1$, hence we also get the boundedness of $T$ from $L^{p_{1}} \times \cdots \times L^{p_{m}}$ into $L^{p}$ as long as $1<p, p_{1}, \ldots, p_{m}<\infty, 1 / p_{1}+\cdots+1 / p_{m}=1 / p$. 
Remark 3. Trivial embeddings between Triebel-Lizorkin spaces allow us to sharpen the main results to obtain boundedness from $\dot{F}_{p_{1}}^{0, s_{1}} \times \cdots \times \dot{F}_{p_{m}}^{0, s_{m}}$ into $\dot{F}_{p}^{0, \min \left(s_{1}, \ldots, s_{m}\right)}$, where $1<p, p_{1}, \ldots, p_{m}<\infty, 1 / p_{1}+\cdots+1 / p_{m}=1 / p$, and $1<s_{1}, \ldots, s_{m} \leq \infty$.

\section{Applications}

I. Let us consider the class of $m$-linear multiplier operators (a priori defined on products of Schwartz function spaces)

$$
\begin{aligned}
T_{\sigma}\left(f_{1}, \ldots, f_{m}\right)(x) & =\int_{\left(\mathbb{R}^{n}\right)^{m}} \sigma\left(\xi_{1}, \ldots, \xi_{m}\right) \widehat{f}\left(\xi_{1}\right) \cdots \widehat{f}\left(\xi_{m}\right) e^{i x \cdot\left(\xi_{1}+\cdots+\xi_{m}\right)} d \xi_{1} \cdots d \xi_{m},
\end{aligned}
$$

with Fourier multipliers $\sigma\left(\xi_{1}, \ldots, \xi_{m}\right)$ satisfying Marcinkiewicz type estimates

$$
\left|\partial_{\xi_{1}}^{\beta_{1}} \ldots \partial_{\xi_{m}}^{\beta_{m}} \sigma\left(\xi_{1}, \ldots, \xi_{m}\right)\right| \leq C_{\beta_{1} \ldots \beta_{m}}\left|\xi_{1}\right|^{-\left|\beta_{1}\right|} \ldots\left|\xi_{m}\right|^{-\left|\beta_{m}\right|}
$$

for all nonzero $\xi_{1}, \ldots, \xi_{m}$ in $\mathbb{R}^{n}$ and all multiindices $\beta_{1}, \ldots, \beta_{m}$. A result of Grafakos and Kalton [6] shows that, in general, Marcinkiewicz multilinear multipliers do not give rise to bounded operators on products of, say, Lebesgue spaces.

Therefore, to achieve boundedness one needs to impose further conditions on the symbol. For example, a slight logarithmic modification of condition (22) as in [6] will do. A different approach to the problem is via almost diagonal estimates [7]. A simple argument using integration by parts implies that, for any family of wavelets $\left\{\phi_{\mu l}\right\},\left\{T_{\sigma}\left(\phi_{\mu_{1} l_{1}}, \ldots, \phi_{\mu_{m} l_{m}}\right)\right\}$ satisfies the size and smoothness conditions (6) needed to make it a family of $m$-linear $\infty$-smooth molecules. Hence, if we further impose the cancellation condition (7), that is,

$$
\int_{\mathbb{R}^{n}} T_{\sigma}\left(\phi_{\mu_{1} l_{1}}, \ldots, \phi_{\mu_{m} l_{m}}\right)(x) d x=0
$$

we do obtain a family of $m$-linear $\infty$-smooth molecules. An easy computation shows that the cancellation condition above is equivalent to

$$
\sigma\left(\xi_{1}, \ldots, \xi_{m}\right)=0
$$

for all $\left(\xi_{1}, \ldots, \xi_{m}\right)$ in the hyperplane $\Gamma:=\left\{\left(\xi_{1}, \ldots, \xi_{m}\right): \xi_{1}+\cdots+\xi_{m}=0\right\}$. Recall that the multiplier of the $j$ th formal transpose $T_{\sigma}^{* j}$ is given by $\sigma^{* j}\left(\xi_{1}, \ldots, \xi_{m}\right)=\sigma\left(\xi_{1}, \ldots, \xi_{j-1},-\xi_{1}-\cdots-\xi_{m}, \xi_{j+1}, \ldots, \xi_{m}\right)$. Therefore, $m$ additional appropriate cancelation conditions on $\sigma$ are required in order to make all the families $\left\{T^{* j}\left(\phi_{\mu_{1} l_{1}}, \ldots, \phi_{\mu_{m} l_{m}}\right)\right\}$ into families of $m$-linear $\infty$-smooth molecules. If we combine all these facts with Theorem 2 we obtain the following 
Proposition 1. Assume that the Fourier multipliers $\sigma, \sigma^{* 1}, \ldots, \sigma^{* m}$ satisfy condition (22) and the following cancellation conditions:

$$
\sigma\left(\xi_{1}, \ldots, \xi_{m}\right)=0, \quad\left(\xi_{1}, \ldots, \xi_{m}\right) \in \Gamma
$$

and

$$
\sigma\left(\xi_{1}, \ldots, \xi_{j-1}, 0, \xi_{j+1}, \ldots, \xi_{m}\right)=0 \quad \forall j, \xi_{j} .
$$

Then $T_{\sigma}$ can be extended as a bounded operator from $\dot{F}_{p_{1}}^{0, s_{1}} \times \cdots \times \dot{F}_{p_{m}}^{0, s_{m}}$ into $\dot{F}_{p}^{0, s}$ for $1<p, p_{1}, \ldots, p_{m}<\infty, 1 / p_{1}+\cdots+1 / p_{m}=1 / p, 1 \leq s<\infty$, $1<s_{1}, \ldots, s_{m} \leq \infty$ and $1 / s_{1}+\cdots+1 / s_{m}=1 / s$.

II. The previous application suggests that one might be able to prove a reduced $T 1$ theorem in the context of Triebel-Lizorkin spaces for a larger class of operators. Indeed, employing certain atomic decomposition techniques, it was proved in [1] that this is the case for bilinear Calderón-Zygmund operators. For different versions of multilinear $T 1$ theorems for $L^{p}$ spaces, see the works of Christ and Journé [2] and Grafakos and Torres [8]. Let us consider the class of m-linear Calderón-Zygmund operators, that is,

$$
T\left(f_{1}, \ldots, f_{m}\right)(x)=\int_{\left(\mathbb{R}^{n}\right)^{m}} K\left(x, y_{1}, \ldots, y_{m}\right) f_{1}\left(y_{1}\right) \cdots f_{m}\left(y_{m}\right) d y_{1} \cdots d y_{m}
$$

whenever $f_{1}, \ldots, f_{m} \in \mathcal{D}\left(\mathbb{R}^{n}\right)$ and $x \notin \bigcap_{j=1}^{m} \operatorname{supp}\left(f_{j}\right)$, and where the kernel $K$ is some $C^{\infty}$ function defined away from the diagonal $x=y_{1}=\ldots=y_{m}$ that satisfies the size and smoothness estimates

$$
\left|D^{\gamma} K\left(x, y_{1}, \ldots, y_{m}\right)\right| \leq \frac{C_{\gamma}}{\left(\left|x-y_{1}\right|+\cdots+\left|x-y_{m}\right|\right)^{m n+|\gamma|}}
$$

for all multiindices $\gamma=\left(\gamma_{1}, \ldots, \gamma_{m+1}\right) \in\left(\mathbb{Z}^{n}\right)^{m+1}$ and all $\left(x, y_{1}, \ldots, y_{m}\right)$ with $x \neq y_{j}$ for some $j$. One could replace the estimates above with more general Lipschitz type conditions on $K$; see, for example, [8].

Let $N$ be a fixed positive integer (the exact value of $N$ is not relevant and will not be made explicit in what follows). Following Stein [9], we say that a $C^{\infty}$ function $\phi$ supported in the unit ball $B_{1}(0)$ of $\mathbb{R}^{n}$ and satisfying

$$
\left\|D^{\alpha} \phi\right\|_{L^{\infty}} \leq 1
$$

for all multi-indices $|\alpha| \leq N$, is a normalized bump. If $f$ and $F$ are functions defined in $\mathbb{R}^{n}$ and $\mathbb{R}^{n} \times \cdots \times \mathbb{R}^{n}$ respectively, we let

$$
f^{w, t}(x)=f\left(\frac{x-w}{t}\right), \quad F^{w, t}\left(x_{1}, \ldots, x_{m}\right)=F\left(\frac{x_{1}-w}{t}, \ldots, \frac{x_{m}-w}{t}\right) .
$$

We say that a continuous $m$-linear operator $T: \mathcal{D} \times \cdots \times \mathcal{D} \rightarrow \mathcal{D}^{\prime}$ satisfies the Multilinear Weak Boundedness Property, or (MWBP), if there exists a positive constant $C$ such that for any $m$ normalized bumps $f_{1}, \ldots, f_{m}$ and any $g \in \mathcal{D}$ supported in $B_{1}(0)$, all $w \in \mathbb{R}^{n}$ and all $t>0$,

$$
\left|\left\langle T^{* i}\left(f_{1}^{w, t}, \ldots, f_{m}^{w, t}\right), g^{w, t}\right\rangle\right| \leq C t^{n}\|g\|_{L^{\infty}}, \quad i=1, \ldots, m .
$$


By using similar methods to the ones outlined in [1], it should be possible to extend the main results there and obtain a reduced multilinear $T 1$ theorem for Calderón-Zygmund operators of the form:

If $T$ is an m-linear Calderón-Zygmund operator satisfying (MWBP) and some appropriate first order cancellation conditions, then $T$ can be extended as a bounded operator $\dot{F}_{p_{1}}^{0, s_{1}} \times \cdots \times \dot{F}_{p_{m}}^{0, s_{m}} \rightarrow \dot{F}_{p}^{0, s}$.

The details of the proof of this claim are left to the interested reader. We also remark that variants of the results above on more general TriebelLizorkin spaces $\dot{F}_{p}^{\alpha, s}$ are possible if we allow for higher order cancellations.

Acknowledgments. The authors would like to thank Rodolfo H. Torres for many valuable suggestions that improved the quality of this presentation, and to the referee for several useful comments.

\section{References}

[1] Á. Bényi, Bilinear singular integral operators, smooth atoms and molecules, J. Fourier Anal. Appl. 9 (2003), 301-319.

[2] M. Christ and J.-L. Journé, Polynomial growth estimates for multilinear singular integral operators, Acta Math. 159 (1987), 51-80.

[3] C. Fefferman and E. M. Stein, Some maximal inequalities, Amer. J. Math. 93 (1971), $107-115$.

[4] M. Frazier and B. Jawerth, A discrete transform and decompositions of distribution spaces, J. Funct. Anal. 93 (1990), 34-170.

[5] M. Frazier, B. Jawerth and G. Weiss, Littlewood-Paley Theory and the Study of Function Spaces, CBMS Regional Conf. Ser. in Math. 79, Amer. Math. Soc., Providence, RI, 1991.

[6] L. Grafakos and N. Kalton, The Marcinkiewicz multiplier condition for bilinear operators, Studia Math. 146 (2001), 115-156.

[7] L. Grafakos and R. H. Torres, Discrete decompositions for bilinear operators and almost diagonal conditions, Trans. Amer. Math. Soc. 354 (2002), 1153-1176.

[8] —, - Multilinear Calderón-Zygmund theory, Adv. Math. 165 (2002), 124-164.

[9] E. M. Stein, Harmonic Analysis: Real-Variable Methods, Orthogonality, and Oscillatory Integrals, Princeton Univ. Press, Princeton, NJ, 1993.

[10] H. Triebel, Theory of Function Spaces, Monogr. Math. 78, Birkhäuser, Basel, 1983.

Department of Mathematics and Statistics

Lederle GRT

University of Massachusetts

Amherst, MA 01003, U.S.A.

E-mail: benyi@math.umass.edu

tzirakis@math.umass.edu 